\title{
New Quantum Theory Explains All the Mysterious Quantum Phenomena
}

\author{
Narendra Swarup Agarwal \\ Independent Researcher, Gurgaon, India \\ Email: nsagarwal@gmail.com
}

How to cite this paper: Agarwal, N.S. (2016) New Quantum Theory Explains All the Mysterious Quantum Phenomena. Journal of Modern Physics, 7, 2135-2154.

http://dx.doi.org/10.4236/jmp.2016.715186

Received: October 4, 2016

Accepted: November 18, 2016

Published: November 21, 2016

Copyright $\odot 2016$ by author and Scientific Research Publishing Inc. This work is licensed under the Creative Commons Attribution International License (CC BY 4.0).

http://creativecommons.org/licenses/by/4.0/

\section{(c) (i) Open Access}

\begin{abstract}
The photons \& the quantum particles display several mysterious Quantum Phenomena which could not be explained earlier. The New Quantum Theory explains not only the long pending Wave Particle Duality but also all other mysterious Quantum Phenomena. The New Quantum Theory is based on the analogy of the atoms \& the solar system and not only supplements but also completes the Quantum Theory. This work explains "how \& why" a photon, an electron or quanta generates the electric \& the magnetic fields of continuously varying intensities \& in the changing directions to form a 3-D Electromagnetic wave. The electromagnetic fields generated by the photons/quantum particles interact with the electromagnetic fields of the mediums (conductor, semiconductor or insulator etc.) to display several mysterious Quantum Phenomena. These interactions are strong enough to change the spin, wavelength, amplitude \& direction of the quantum particles in the medium. The spinorbit interlocking/interactions, transverse spin, vortex spin texture \& evanescent waves etc. are the results of such interactions. This work explains some of the quantum phenomena in brief so that the various Quantum Phenomena can be modified \& developed for the different applications.
\end{abstract}

\section{Keywords}

Quantum Phenomena, Off-Centre Nucleus, Mass-Frequency Constant, True Mass of Photon \& 3-Dimensional Electromagnetic Waves

\section{Introduction}

About 350 years ago, Newton proposed that the light was made up of small discrete particles moving in a straight line having kinetic energy while Christian Huygens proposed the Wave Theory that the light was made up of waves vibrating up and down perpendicular to the direction of the light travelled. Only after more than a century in 
1801, the double slit experiment by Thomas Young proved the dual nature of the light, travelling as waves but being absorbed as the particles. Since the year 1801, the dual nature of light remains a puzzle for the scientists and could not be explained even by the Quantum Theory.

The photons of the electromagnetic radiations are the particles, but always travel as waves. The Quantum Theory developed in the $20^{\text {th }}$ century accepted the Wave Particle Duality in absence of a solution. However, Einstein [1] did not accept the Wave Particle Duality and wrote:

"This double nature of radiation (and of material corpuscles) is a major property of reality, which has been interpreted in quantum mechanics in an ingenious and amazingly successful fashion. This interpretation, which is looked upon essentially as final by almost all contemporary physicists appears to me as only a temporary way out".

Not only the Wave Particle Duality, but almost all other Quantum Phenomena like Interference, Polarization, Refraction, Formation of the Electric/Magnetic fields \& 3-D Electromagnetic Waves, Quantum Tunneling and Quantum Spin Hall Effect etc. also could not be explained by the Quantum Theory. It is necessary to supplement the Quantum Theory so that all the mysterious Quantum Phenomena (already known or to be discovered in future) can be explained and used for the different applications.

The "New Quantum Theory" [2], developed in the year 2012, explains all the Quantum Phenomena. This theory is based on the analogy of the atoms \& the solar system, the realities of the nature.

The nucleus of a tiny atom has $>99.9 \%$ mass of the atom, but occupies only about $10^{-10}$ times the volume of the whole atom. Similarly, the Sun as the nucleus of the solar system, has $>99.86 \%$ mass of the solar system and occupies only about $10^{-10}$ times the volume of the whole solar system. The nuclei of both the atoms \& as well as the solar system are not in the centre, but located off-centre.

The New Quantum Theory [2] explicates " $A$ photon or quanta has a nucleus of the mass \& the charge located off-center, similar to the nucleus of an atom".

The structure of a photon, based on the New Quantum Theory, is shown in Figure 1. The off-centre location of a nucleus of the mass in a spinning particle bestows special characteristics to the particle. Such location of mass in a photon develops intrinsic spin angular momentum and moves the photon like a wave. This characteristic explains the Wave Particle Duality, Interference \& Polarization phenomena etc.

The deflection of the polarized photons on reflection from their straight line path proves the mass in the photons. The increase in the deflection of the polarized photons, on increasing the angle of polarization, proves that the mass in the photons is located off-centre. If a photon has absolutely zero mass, it can never travel as a wave. This work proves that the mass of a photon is directly proportional to the frequency of the photon by an equation $m_{t}=a f$, similar to the equation $E=h f$. The value of the new constant "a" (Mass-Frequency Constant) is $1.474499440284 \times 10^{-50} \mathrm{~kg} \mathrm{sec}$. The photons of the different frequencies have different masses, similar to the different masses of the different atoms. The isotopes of an atom also have different masses. 


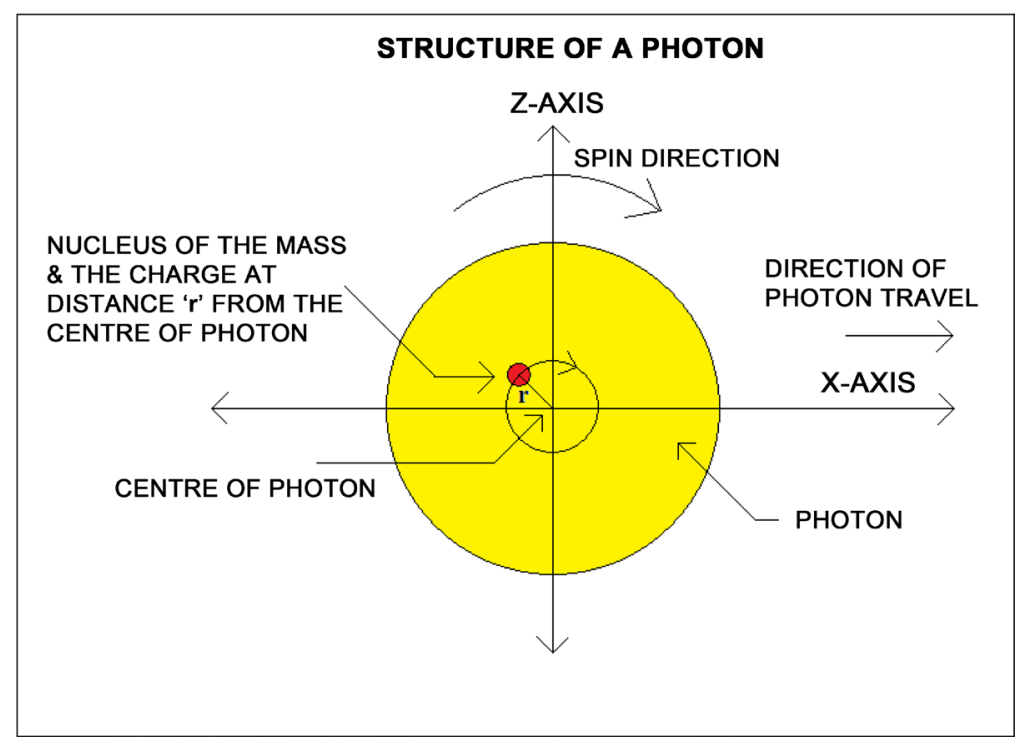

Figure 1. Yellow spherical particle shows a photon. Small red sphere is the "nucleus of the mass \& the charge", which rotates continuously around the centre of the photon in a circle of radius " $r$ " with the spin of the photon. This photon is spinning in a clockwise direction and moves in the X-direction.

Similarly, the charge along with the mass in the nucleus located off-centre in a spinning photon generates the intrinsic spin magnetic dipole moment and the electric \& the magnetic fields of continuously varying intensities \& directions. A moving charge generates the electric \& the magnetic fields. If there is no charge in the photon, it cannot generate any electric or the magnetic field.

This work explains in detail "How \& Why?" the charge of a spinning photon travelling as a wave, generates the electric \& the magnetic fields of continuously varying intensities in the changing directions to form a correct 3-D electromagnetic wave for the first time.

The electric \& the magnetic fields/intrinsic spin magnetic dipole moment generated by the photons/quantum particles interact with the electromagnetic fields of the different mediums (conductor, semiconductor or insulator etc.) to display the mysterious quantum phenomena. These interactions develop the electromagnetic force to changel modify the spin, wavelength, amplitude \& direction of the quantum particles in the medium. The spin-orbit interlocking/interactions, transverse spin, vortex spin texture \& evanescent waves etc. are the results of such interactions. The New Quantum Theory can explain all the mysterious Quantum Phenomena.

This work explains some of the Quantum Phenomena in brief, to develop \& modify the Quantum Phenomena for the new developments/improvements in the medical, industrial \& commercial applications for the benefit of all.

\section{Mass \& Energy of Photon}

The most important discoveries in the physics include: Planck' Postulation "Energy of radiation can only be emitted in discrete packets or quanta, i.e. in multiples of the 
energy $E=n h f$. where $n$ is an integer' and Einstein" Assumption "The emission or transformation of light are more readily understood if one assumes that the energy of light is discontinuously distributed in space".

Based on the above discoveries-the energy exists in the form of discrete quanta and all these quanta are not continuous but separate.

According to the original equation of Einstein of mass-energy equivalence $e=m c^{2}$, all the $100 \%$ mass can be coverted to energy, but it's not true. Therefore, subsequently the equation was revised to $e=\sqrt{(p c)^{2}+\left(m c^{2}\right)^{2}}$. All the mass cannot be converted to the energy; some mass will always be left out with the energy.

- No form of energy can exist in isolation without any object/matter.

- An object/ matter (of mass) is required to contain the energy.

- A photon is a discrete particle of energy.

- A photon has not only the energy but being a discrete particle or object/matter, it also has mass.

An absolute vacuum with no matter or mass has no energy.

A photon is a particle having mass which moves \& spins by virtue of its energy.

"Wave Particle Duality \& Interference Explained" [4] explains the mass of the photons and also introduces a new term "True Mass of Photon" (actual mass of the photon) which is always constant in all the conditions, irrespective of the velocity of the photon or the position of the observer etc. (Rest mass, Relativistic mass \& Invariant mass etc. are all the conditional terms of the mass).

\section{Some Proofs of the Mass in the Photons}

According to the New Quantum Theory, the mass in a photon is located off-centre, as shown in Figure 1. The mass of the spinning photon generates spin angular momentum in the plane of the spin of the photon. This spin angular momentum is in addition to the linear momentum of the photon. The spin angular momentum, due to the mass of the photon, is responsible for the mysterious behavior of the photons to exhibit various Quantum Phenomena.

1) Experimental Proof of Mass in Photon: The experiments of reflection of the polarized photons, based on Newton's law of Inertia, prove the presence of the mass located off-centre in the photons. The polarized photons deflect on reflection by a plain surface coated mirror due to the off-centre mass of the photon from their straight line path after reflection. The spin angular momentum deviates the photons by developing the inertial force at the contact point of the mirror. The details of the experiments are given in "Experimental Proof of Mass in Photon" [3].

The experiment confirms the followings:

a) A photon has mass. The mass in the photon generates an inertial force at the contact point of the mirror to deflect the photons from their straight-line path on reflection.

b) The mass of a photon is not in the centre or uniformly distributed but is located off-centre in the photon. The inertial force, developed at the contact point of the 
mirror, varies with the angle of polarization of the photon.

c) The increase in the frequency of the photons increases the deflection of the photons on reflection. High frequency of the photons develops high spin angular momentum resulting in higher inertial force at the contact point of the mirror and increased deflection.

d) A photon is a spherical particle. The photons deflects in the path of a curve by varying the angle of polarization as explained below:

- If the plane of polarization is vertical (X-Z) plane and the photons move in X-direction at an angle about $15^{\circ}$ downward from the horizontal line towards a horizontal plain surface coated mirror, the photons after reflection deflect only downward, in the X-direction. The inertial force developed by the mass of the photons is only in the vertical $(\mathrm{X}-\mathrm{Z})$ plane.

- There is no deflection on either side of the vertical $(X-Z)$ plane. In this case of the $0^{\circ}$ angle of polarization, the mass of the photons spins only in the vertical plane and develops the inertial force in the vertical $(\mathrm{X}-\mathrm{Z})$ plane, to move the photons only downward (Z-direction) on the screen/sensor.

- When the plane of polarization tilts towards the left side of the photon, the inertial force develops in the two planes (X-Y \& X-Z planes), as the mass of a photon spins in an intermediate plane.

- The photon after reflection deflects towards the right side (Y-direction) due to the inertial force in X-Y plane. The deflection of the polarized photons increases by increasing the angle of polarization up to $90^{\circ}$ from the vertical plane.

- Simultaneously, the inertial force in $X-Z$ vertical plane reduces with increase in the angle of polarization up to $90^{\circ}$ from the vertical plane; this moves the photons up in the Z-direction.

- As the angle of polarization of photon increases from $0^{\circ}$ to $90^{\circ}$ (from the vertical plane to the horizontal plane), the photon moves in the path of a curve by deflecting in both the directions-Y direction (right side) \& $\mathrm{Z}$ direction (upwards).

This experiment proves that the photon is a spherical particle and has the mass located off-centre in the photon. The effects of the magnetic field of the earth \& other external factors etc. are constant and negligible in the experiment for small travel of photons of few meters only. Any physics laboratory can perform these experiments. It is advisable to keep the distance between the mirror and the screen/sensor 10 meters or more to note the visible deflections of photons after the reflection.

2) Wave Particle Duality: A photon particle has both the linear momentum as well as the spin angular momentum. For a linearly polarized photon, the direction of the spin angular momentum continuously changes in $360^{\circ}$ with the spin of the photon in the plane of polarization. The combined effect of the spin angular momentum \& the linear momentum develops "Resultant Momentum". The direction \& the magnitude of the Resultant Momentum change continuously and moves the photon particle up \& down during its travel to form a Wave in the plane of polarization.

For a circularly polarized photon, the spin angular momentum continuously changes 
in all the $3 \mathrm{X}, \mathrm{Y} \& \mathrm{Z}$ directions. Therefore, the Resultant Momentum also changes continuously in all the 3 directions to move the photon particle in all the $3 \mathrm{X}, \mathrm{Y} \& \mathrm{Z}$ directions.

3) Polarization Phenomenon: This phenomenon also takes place due to the spin angular momentum created by the mass of the photon. If the continuously changing direction of the spin angular momentum confines only in one plane, the Resultant Momentum remains in the same plane and the photon is linearly polarized and forms wave in this plane.

If the direction of the spin angular momentum of the photon changes continuously in all the $3 \mathrm{X}, \mathrm{Y} \& \mathrm{Z}$ directions, the direction of the Resultant Momentum also change in all the 3 directions. Such a photon is circularly polarized and forms wave in all the 3 planes.

4) Interference Phenomenon: In the Constructive Interference, the two photons are exactly in the same phase. The nuclei of the mass \& the charge of both the photons superimpose to coalesce to form single identity. The strong force attraction is possible between the two photons. This doubles the spin angular momentum to double the amplitude of the photon. There is no change in the wavelength of the photon even after doubling of the linear momentum as the total mass of the combined photons also doubles.

In the Destructive Interference, the two photons are in the opposite phases. Both the photons meet at the centerline and coalesce to form single identity. The strong force attraction may be possible between the two photons. The spin angular momentums of both the photons are in opposite directions, $180^{\circ}$ from each other. Therefore, both the superimposed photons, as one, move together in the centerline only with zero amplitude.

If a photon has absolutely zero mass or the mass is uniformly distributed in the photon or the mass is in the centre of the photon, it can never travel as a wave. Such a photon cannot display any of the quantum phenomena, including the Wave Particle Duality.

Wave Particle Duality \& Interference Explained [4] explains the Wave Particle Duality, Constructive, Destructive \& Intermediate Interferences and Polarization phenomena.

The classical mechanics and the two famous equations of mass-energy equivalence by Albert Einstein $e=m c^{2}$ \& by the Planck-Einstein relation $E=h f$, correlate mass, velocity, momentum, energy $\&$ frequency etc. of the photons.

It is very important to note the difference in the different types of energy in both the above-mentioned equations for the energy.

The energy " $\mathrm{e}$ " in the original equation $e=m c^{2}$, is the energy when the mass of a photon or quanta completely converts to the energy by a nuclear conversion also known as mass-energy equivalence.

Whereas, " $E$ " in the equation $E=h f$, is the sum of all the energy possessed by a photon or quanta as a particle. This energy includes different forms of energy such as 
linear kinetic energy, rotational kinetic energy, potential energy and any other form of the energy. Therefore, the term " $E$ " in the equation $E=h f$ is the total energy possessed by a photon or quanta. This energy " $E$ " does not include the energy produced by the nuclear conversion of the mass of the photon to the energy.

Planck's equation derives a new equation for the "True Mass of photon $\left(m_{t}\right)$ " as under:

(A horizontally moving photon is considered to derive the equation)

The energy of a Photon $(E)$ = Linear Kinetic Energy + Rotational Kinetic Energy

- Linear Kinetic Energy of photon: $\frac{1}{2} m_{t} c^{2}$

- Rotational Kinetic Energy of photon: $\frac{1}{2} I \omega^{2}$

- Total Energy of photon $E=\frac{1}{2} m_{t} c^{2}+\frac{1}{2} I \omega^{2}$

$$
E=h f=\frac{1}{2} m_{t} c^{2}+\frac{1}{2} m_{t} r^{2}(2 \pi f)^{2}
$$

- Or True Mass of Photon

$$
m_{t}=\frac{2 h f}{c^{2}+4 \pi^{2} r^{2} f^{2}}
$$

In the above equation, the term $4 \pi^{2} r^{2} f^{2}$, in the denominator is negligibly small in comparison to the large value of $c^{2}$. Therefore, the equation for the True Mass of Photon simplifies to:

$$
m_{t}=\frac{2 h f}{c^{2}}
$$

- Or

$$
m_{t}=a f
$$

True Mass of a Photon is directly proportional to the frequency of the photon.

Where " $a$ " is constant and called as "Mass-Frequency Constant" for the photons. The value of this constant is given below:

$$
a=\frac{2 h}{c^{2}}=1.474499440284 \times 10^{-50} \mathrm{~kg} \cdot \mathrm{sec}
$$

where

- $m_{i}$ True Mass of photon;

- c. Linear velocity of photon;

- I: Moment of Inertia of photon;

- $\omega$ : Angular velocity of photon;

- $f$ : frequency of photon;

- $r$. Distance of centre of mass of photon from the centre of photon;

- h: Planck's constant;

- a: Mass-Frequency constant;

Notes: 
1. The value of " $r$ " being of the order of $10^{-17}$, the term $4 \pi^{2} r^{2} f^{2}$ is negligibly small in comparison to $c^{2}$ which is of the order of $10^{16}$.

2. The energy of a photon, " $E$ ' includes energy in all the forms of the energy possessed by the photon. This does not include the energy due to the mass-energy conversion $e=m c^{2}$, by the nuclear conversion. The potential energy is not considered here due to no or negligible change in the elevation.

Now "True Mass" of a photon of any frequency can be calculated simply by multiplying the frequency of the photon with "a" (Mass-frequency constant) having value $1.474499440284 \times 10^{-50} \mathrm{~kg} \mathrm{sec}$. The True Masses of few Photons of different wavelengths/frequencies are calculated in Table 1.

\section{Structure of a Photon}

Based on the analogy of the atoms as well as the solar system, the New Quantum Theory [2] conceives the structure of a photon with a small nucleus of the mass and the charge. The nucleus of the photon is not in the centre of the photon, but located off-centre, similar to the atoms \& the solar system. Figure 1 shows the structure of a photon.

\section{Charge of a Photon}

James Clerk Maxwell in the $19^{\text {th }}$ century described how the electric and the magnetic fields are generated by the charges, currents and changes of each other. Maxwell developed the Theory of Electromagnetism and also conceived the formation of an electromagnetic wave by a moving charge in the $19^{\text {th }}$ century itself.

A moving point charge generates the electric \& the magnetic field forming an electromagnetic wave. The intensities of the electric \& the magnetic fields generated by a moving charge depend on the charge, velocity and acceleration of the moving charge.

Table 1. True mass of photon for different wavelength \& frequency.

\begin{tabular}{ccccc}
\hline $\begin{array}{c}\text { Radiation } \\
\text { Type }\end{array}$ & $\begin{array}{c}\text { Wavelength } \\
\text { (Nanometer) }\end{array}$ & $\begin{array}{c}\text { Energy } \\
\text { (Joule) }\end{array}$ & $\begin{array}{c}\text { Frequency }(f) \\
(\text { Cycles/second })\end{array}$ & $\begin{array}{c}\text { True Mass of } \\
\text { Photon }(\mathrm{aff})(\mathrm{Kg})\end{array}$ \\
\hline Gamma Rays & 0.001 & $1.9864458 \times 10^{-13}$ & $2.99792458 \times 10^{20}$ & $4.420438115 \times 10^{-30}$ \\
X Rays & 1.0 & $1.9864458 \times 10^{-16}$ & $2.99792458 \times 10^{17}$ & $4.420438115 \times 10^{-33}$ \\
Ultra Violet & 100 & $1.9864458 \times 10^{-18}$ & $2.99792458 \times 10^{15}$ & $4.420438115 \times 10^{-35}$ \\
Dark Blue & 442 & $4.4942213 \times 10^{-19}$ & $6.78263479 \times 10^{14}$ & $1.000099121 \times 10^{-35}$ \\
Green & 531 & $3.7409525 \times 10^{-19}$ & $5.64580900 \times 10^{14}$ & $8.324742213 \times 10^{-36}$ \\
Red & 633 & $6.6260700 \times 10^{-19}$ & $4.73605778 \times 10^{14}$ & $6.983314558 \times 10^{-36}$ \\
Infra Red & 1000 & $1.9864458 \times 10^{-19}$ & $2.99792458 \times 10^{14}$ & $4.420438115 \times 10^{-36}$ \\
Ultra High Frequency & $1,000,000$ & $1.9864458 \times 10^{-22}$ & $2.99792458 \times 10^{11}$ & $4.420438115 \times 10^{-39}$ \\
Very High Frequency & $1,000,000,000$ & $1.9864458 \times 10^{-25}$ & $2.99792458 \times 10^{8}$ & $4.420438115 \times 10^{-42}$ \\
\hline
\end{tabular}

Note: The mass of a photon of the Gamma Rays is more than the mass of an electron. 
The Electromagnetic radiations generate the photons. A photon always generates the electric \& the magnetic fields in the form of an electromagnetic wave. This is possible only with the presence of the charge in the photons, the charge may be either positive or negative.

Only a moving \& spinning particle having the mass \& the charge located off-centre, can generate the electric \& the magnetic fields of varying intensities and in changing directions to form a 3-D Electromagnetic Wave.

Any moving particle with absolutely zero charge cannot generate any electric or magnetic field and cannot display any quantum phenomena involving the electric or the magnetic field.

A photon has a nucleus of the mass \& the charge located off-centre. The continuously spinning photon with the charge located off-centre develops an intrinsic spin magnetic dipole moment in addition to the electromagnetic field. This intrinsic spin magnetic dipole moment/electromagnetic field of the photons interact with magnetic dipole moments/electromagnetic fields of the external medium to display several mysterious Quantum Phenomena.

No extra proof of the charge in the photon is required.

\section{How \& Why a Photon Form 3-D Electromagnetic Wave?}

A photon generates the electric \& the magnetic fields of continuously varying intensities and in changing directions to form a 3-D Electromagnetic Wave. Following points are explained before starting the formation of an Electromagnetic wave (EM) by a linearly polarized photon:

- Wave Particle Duality \& Interference Explained [4] explains the Wave Particle Duality ("How \& Why" a photon always travels in the path of a wave?)

- The reference point of a photon is the centre of the photon.

- A photon is always spinning; its nucleus of the mass and the charge rotates in a circle around the centre of the photon.

- A moving charge produces the electric \& the magnetic fields depending on the intensity of charge, velocity and acceleration of the moving charge particle. The intensities of the electric \& the magnetic fields increase with the increase in velocity of the charge. If the velocity of the charge decreases, the intensities of both the electric \& the magnetic fields decrease.

- In the upper half cycle of EM wave formation, the photon spins from $0^{\circ}$ to $180^{\circ}$. The nucleus of the photon moves in the forward direction with respect to the centre of the photon (reference point) as shown by points $\mathrm{P} \rightarrow \mathrm{Q} \rightarrow \mathrm{R}$ in Figure 2.

- In the lower half cycle of EM wave formation, the photon spins from $180^{\circ}$ to $360^{\circ}$. The nucleus of the photon moves in the backward direction with respect to the centre of the photon (reference point) as shown by points $R \rightarrow S \rightarrow P$ in Figure 2 .

- Even though the nucleus of the photon is always rotating at constant angular velocity around the centre, the forward horizontal movement of the charge of the nucleus 
is the fastest at point " $Q$ " (90 spin position of the photon). Similarly, the backward horizontal movement of the charge in the nucleus is the fastest at point " $S$ " $\left(270^{\circ}\right.$ spin position of the photon). Therefore, the intensities of both the electric as well as the magnetic fields are the maximum at both these points $\mathrm{Q} \& \mathrm{~S}$.

- The horizontal forward or backward movements of the charge with respect to the centre of the photon are zero at points " $P$ " \& “ $R$ " $\left(0^{\circ} \& 180^{\circ}\right.$ respectively $)$ on the centreline of photon wave.

Therefore, the intensities of both the electric as well as the magnetic fields are zero at both these points $\mathrm{P} \& \mathrm{R}$ and no electric or magnetic fields generate.

- The movement of the charge of the nucleus in the forward direction, with respect to the centre of the photon, generates a positive electric field and an outward magnetic field. Similarly, the movement of the charge of the nucleus in the backward direction, with respect to the centre of the photon, generates a negative electric field and an inward magnetic field.

To explain the formation of EM wave, consider a linearly polarized photon in the vertical plane (X-Z), travelling horizontally in X-Axis. Z-Axis is the vertical axis and the $\mathrm{Y}$-Axis is perpendicular to the paper/laptop screen. The photon starts from the centreline in X-Axis from the $0^{\circ}$ spin position of the nucleus in the photon.

\section{Formation of the Electric Field Wave}

1) As the photon spins from $0^{\circ}$ to $180^{\circ}$, it moves in the upper half cycle of the wave path in the X-Z plane.

2) The charge in the nucleus of photon moves in the forward horizontal direction with respect to the centre of photon as shown in Figure 2 from "P" to " $Q$ " to " $R$ ".

3) At point "P", the horizontal forward movement of the charge in the nucleus of the photon is zero with respect to the center of the photon; the intensity of the electric field is also zero at point " $\mathrm{P}$ " ( $0^{\circ}$ spin position of photon).

4) As the photon spins from $0^{\circ}$ to $90^{\circ}$, there is a continuous acceleration in the forward horizontal velocity of the charge in the forward direction with respect to the centre of the photon. Therefore, the intensity of the positive electric field continuously increases from $0^{\circ}$ spin position of the photon up to the crest position of the photon at point "Q" ( $90^{\circ}$ spin position) as shown in Figure 3.

5) At the crest point of wave ( $90^{\circ}$ spin position of photon), the horizontal forward velocity of the charge with respect to the centre of the photon is the maximum. Therefore, the intensity of the positive electric field is also the maximum at $90^{\circ}$ spin of the photon at the crest position of the photon at point " $Q$ ".

6) As the photon spins further from $90^{\circ}$ spin position, there is continuous retardation in the forward horizontal velocity of the charge with respect to the centre of the photon. Therefore, the intensity of the positive electric field continuously decreases from the crest position of photon up to the point " $R$ " ( $180^{\circ}$ spin position) as shown in Figure 3. 
ENLARGED VIEW OF THE CENTRE \& THE NUCLEUS OF A PHOTON

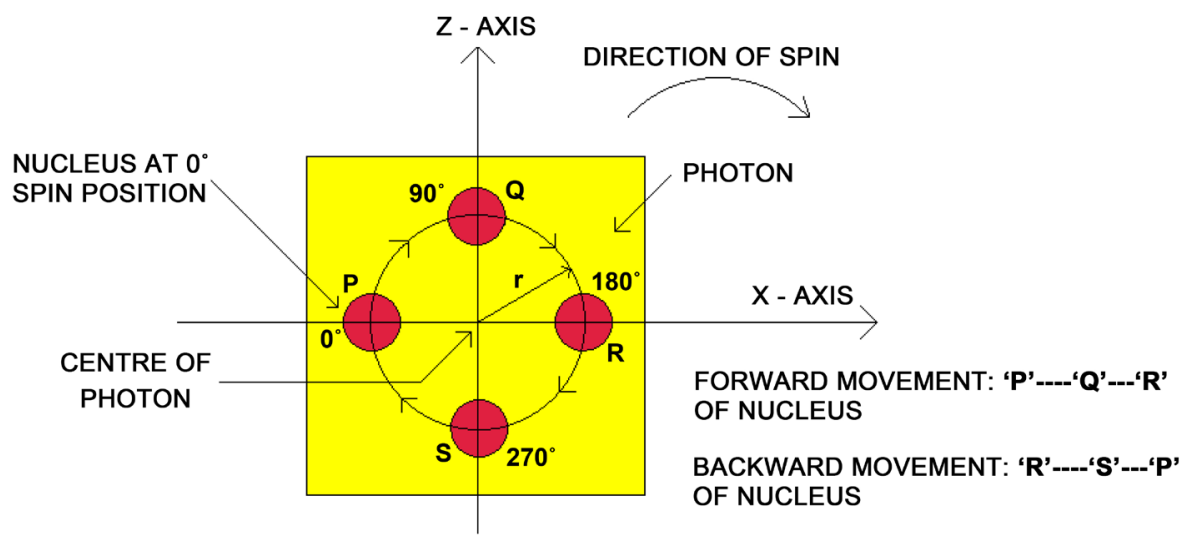

Figure 2. The nucleus of a photon circles the centre of the photon with the spin of the photon. During first half $\operatorname{spin}\left(0^{\circ}\right.$ to $\left.180^{\circ}\right)$, the nucleus moves from point " $\mathrm{P}$ " to "Q" to " $\mathrm{R}$ " in the forward horizontal direction with respect to the centre of the photon. During second half spin $\left(180^{\circ}\right.$ to $360^{\circ}$ ), the nucleus moves from point " $\mathrm{R}$ " to " $\mathrm{S}$ " to " $\mathrm{P}$ " in the backward horizontal direction with respect to the centre of the photon.

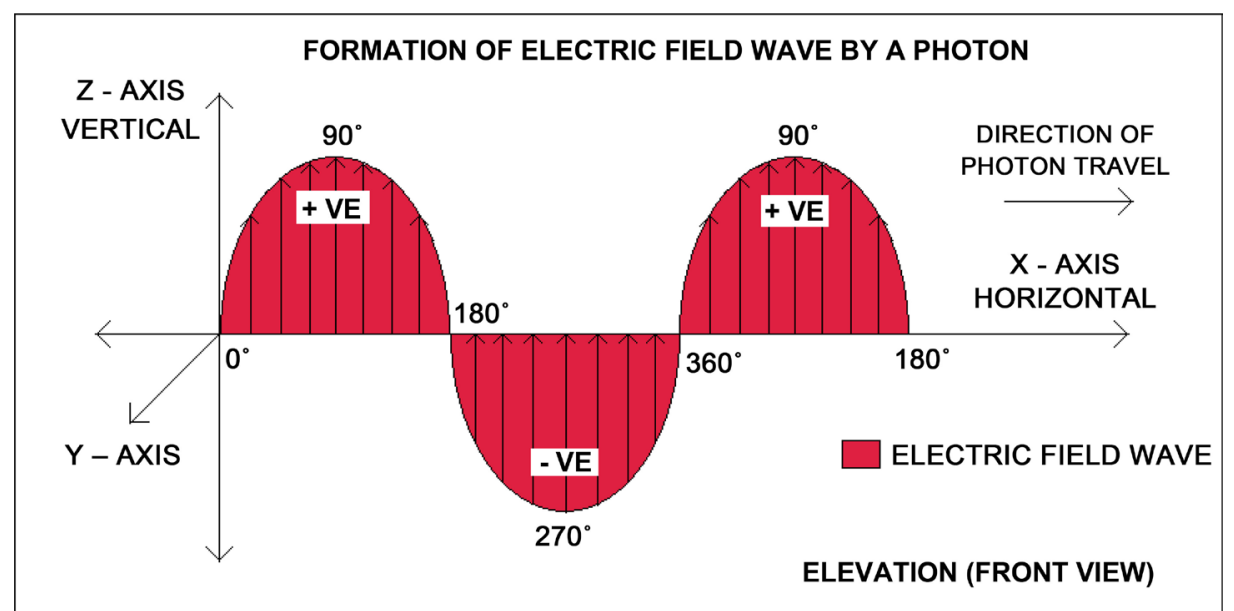

Figure 3. Front view of the electric field wave. As the charge in the nucleus of photon moves in the forward horizontal direction, (+VE) electric field wave generates. The backward horizontal movement of the charge generates $(-\mathrm{VE})$ electric field wave. The intensity of the electric field is proportional to the horizontal velocity of the charge with respect to the centre of the photon.

7) At the point " $R$ " of the charge in the nucleus, the horizontal movement of the charge with respect to the centre of the photon is zero; therefore the intensity of electric field is also the zero at $180^{\circ}$ spin position of the photon.

8) As the photon spins further from $180^{\circ}$, lower half cycle of the electric field wave is formed. During the spin of the photon from $180^{\circ}$ to $360^{\circ}$, the charge in the nucleus moves from " $\mathrm{R}$ " to " $\mathrm{S}$ " to " $\mathrm{P}$ " in the backward horizontal direction with respect to the centre of the photon as shown in Figure 2.

9) With the spin of the photon from $180^{\circ}$, there is continuous acceleration in the 
backward horizontal velocity of the charge with respect to the centre of the photon. Therefore the intensity of the negative electric field continuously increases from the point " $R$ " ( $180^{\circ}$ spin position of the photon) up to the trough position of the photon at point "S" ( $270^{\circ}$ spin position of the photon).

10) At the trough position of the wave ( $270^{\circ}$ spin position of the photon), the horizontal backward velocity of the charge with respect to the centre of the photon is the maximum. Therefore, the intensity of the negative electric field is also the maximum at $270^{\circ}$ spin position of the photon.

11) As the photon spins further from $270^{\circ}$ spin position, there is continuous retardation in the backward horizontal velocity of the charge with respect to the centre of the photon. Therefore, the intensity of the negative electric field continuously decreases from the trough position of photon point "S" ( $270^{\circ}$ spin position) up to the point "P" (360 spin position of photon) (the end of the first wave cycle \& the start of the next wave cycle).

12) At the point "P" of the charge (end of the first wave cycle \& the start of next wave cycle), the horizontal velocity of the charge is zero, therefore the intensity of the electric field is also the zero at $360^{\circ}$ spin position of the photon.

13) During $0^{\circ}$ to $180^{\circ}$ spin of the photon, the charge is moving in the forward direction with respect to the centre of photon, therefore the positive electric field generates. Similarly, during $180^{\circ}$ to $360^{\circ}$ spin of the photon, the charge is moving in the backward direction with respect to the centre of the photon, therefore the negative electric field generates.

14)This explains the formation of the electric field wave of continuously varying intensity of one full cycle from $0^{\circ}$ to $360^{\circ}$ spin of the photon in both the directions of the centreline (X-Axis) in X-Z plane by a photon as shown in Figure 3.

\section{Formation of Magnetic Field Wave}

1) As the photon spins from $0^{\circ}$ to $180^{\circ}$, the charge in the nucleus has forward movement with respect to the centre of the photon. This forward movement of the charge generates a magnetic field in all the $3 \mathrm{X}, \mathrm{Y} \& \mathrm{Z}$ planes which is always perpendicular to the plane of the electric field (X-Z plane).

2) As there is a continuous acceleration in the forward horizontal velocity of the charge from point "P" to the crest point "Q" ( $0^{\circ}$ to $90^{\circ}$ spin of photon), the intensity of the outward magnetic field increases continuously from $0^{\circ}$ to $90^{\circ}$ spin of the photon. Similarly, there is continuous retardation in the forward horizontal velocity of the charge from point " $Q$ " to the point " $\mathrm{R}$ " ( $90^{\circ}$ to $180^{\circ}$ spin of photon), the intensity of the outward magnetic field decreases continuously.

3) At the crest point of the wave ( $90^{\circ}$ spin position of photon), the horizontal forward movement of the charge with respect to the centre of the photon is the maximum. Therefore, the intensity of the outward magnetic field is also the maximum at crest point "Q" (90 spin of photon).

4) At the point "R" of the nucleus, the horizontal velocity of the charge is zero, there- 
fore the intensity of the magnetic field is the zero on the point " $R$ " $\left(180^{\circ}\right.$ spin position of the photon) and no magnetic field generates.

5) With the spin of the photon from $0^{\circ}$ to $180^{\circ}$, the outward magnetic field generates as 3-Dimensional curve projecting out from the upper half cycle of the photon wave path in the $+Y$-direction. The magnetic field is also curved due to the continuous variation in the intensity of the magnetic field as shown in Isometric view of Electromagnetic wave in Figure 4.

6) The photon forms the second half cycle of the magnetic wave during its spin from $180^{\circ}$ to $360^{\circ}$. During this spin, the charge in the nucleus moves in the backward horizontal direction with respect to the centre of the photon, generating the inward magnetic field wave in the (-)Y-direction while the photon generates the lower half cycle of EM wave.

7) As there is a continuous acceleration in the backward horizontal velocity of the charge from point " $\mathrm{R}$ " to the trough point " $\mathrm{S}$ " ( $180^{\circ}$ to $270^{\circ}$ spin of the photon), the intensity of the inward magnetic field increases continuously from $180^{\circ}$ to $270^{\circ}$ spin of the photon. Similarly, there is a continuous retardation in the backward horizontal velocity of the charge from the point "S" to the point " $\mathrm{P}$ " $\left(270^{\circ}\right.$ to $360^{\circ}$ spin of the photon), the intensity of the inward magnetic field decreases continuously.

8) At the trough point " $S$ " of the wave ( $270^{\circ}$ spin position of the photon), the horizontal backward movement of the charge with respect to the centre of the photon is the

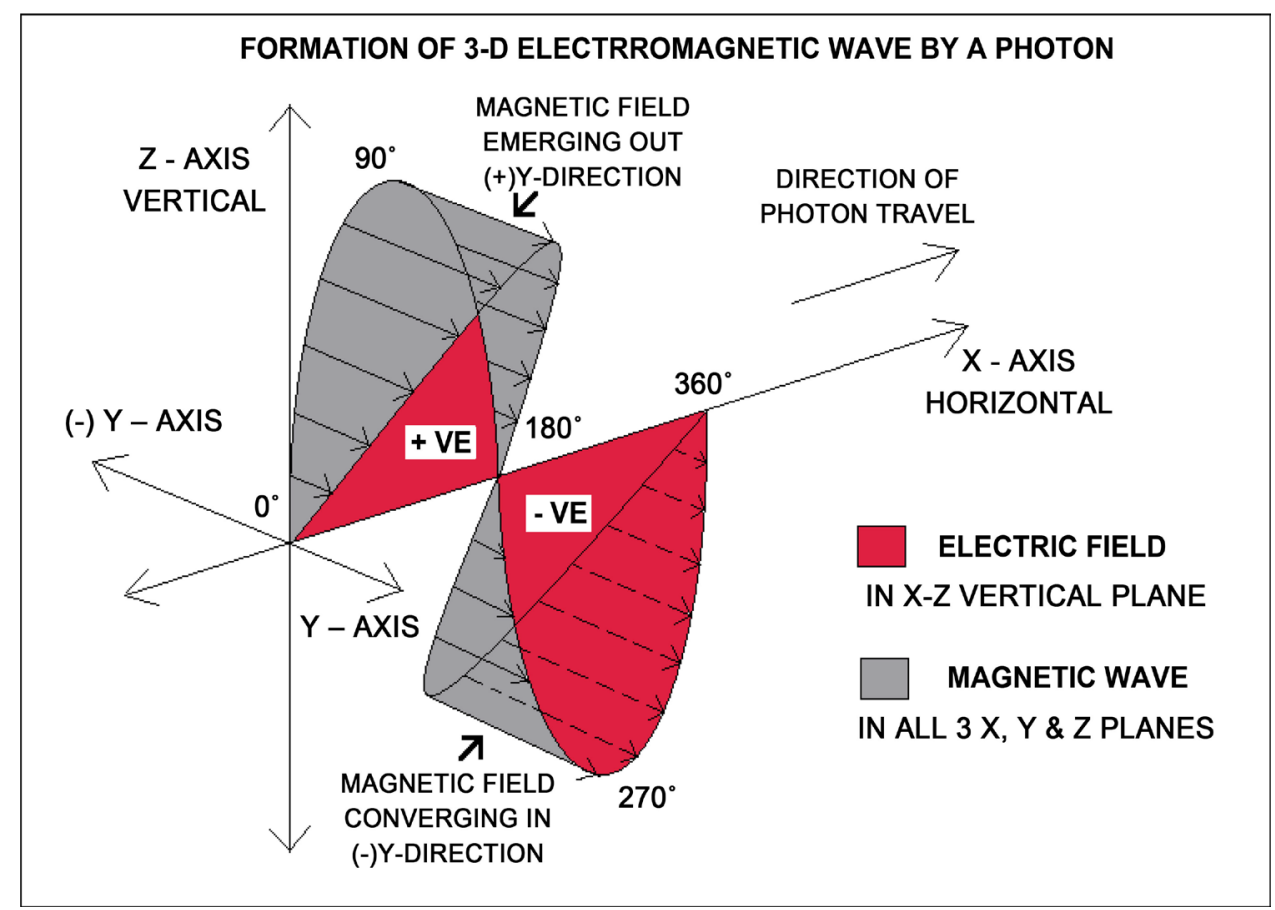

Figure 4. Isometric view of the 3-D electromagnetic wave. The photon is polarized in X-Z plane, therefore the charge in the nucleus of the photon generates the electric field wave in the $X-Z$ plane only. The charge generates the magnetic field wave from the location of the charge in the wave and not from the centreline. The magnetic field generates in the direction perpendicular to the electric field. Therefore, the magnetic field wave generates in the 3-dimensions. 
maximum. This generates the inward magnetic field of the maximum intensity at $270^{\circ}$ spin of photon.

9) At the point "P" of the charge (the end of the first wave cycle \& the start of the next wave cycle), the horizontal movement of the charge is zero, therefore the intensity of the magnetic field is zero at the point " $\mathrm{P}$ " ( $360^{\circ}$ spin position of the photon).

10) During $0^{\circ}$ to $180^{\circ}$ spin of a photon, the charge is moving in the forward horizontal direction with respect to the centre of the photon, therefore the outward magnetic field generates. Similarly, during $180^{\circ}$ to $360^{\circ}$ spin of the photon, the charge is moving in the backward direction with respect to the centre of the photon, therefore the inward magnetic field generates.

11)This explains the formation of the magnetic field wave of continuously varying intensity of one full cycle from $0^{\circ}$ to $360^{\circ}$ spin of the photon in both the directions of the centreline (X-Axis) in X-Y-Z planes by a photon as shown in Isometric view of Electromagnetic wave in Figure 4.

12)The above explains "how \& why" a linearly polarized photon forms a 3-D Electromagnetic Wave. Whereas, the electric field is only in X-Z plane for a linearly polarized photon, the magnetic field is formed in all the $3 \mathrm{X}, \mathrm{Y} \& \mathrm{Z}$ planes. The magnetic field is perpendicular to the electric field.

For a circularly polarized photon, the electrical field wave is formed in all the 3-directions $\mathrm{X}, \mathrm{Y} \& \mathrm{Z}$ like the magnetic field wave in all the 3-directions. Both the electric \& the magnetic fields are perpendicular to the each other.

The magnetic field generates from the location of the charge in the photon and not from the centreline of the photon wave. For example, if the amplitude of an electromagnetic wave is 5 meters, the photon at the crest position is 5 meters above the centreline. The charge in the nucleus of the photon generates the maximum magnetic field wave 5 meters above the centreline from the position of the charge in the direction perpendicular to the electric field and not from the centreline.

The electromagnetic fields of the photons/quantum particles interact with the electromagnetic field of the medium or other objects to display several mysterious Quantum Phenomena.

\section{Explanation of Quantum Phenomena}

The mass \& the charge in the nucleus of a photon or a quanta located off-centre, bless the quantum particle with the mysterious characteristics. Only such a photon or quanta can explain Wave Particle Duality, the formation of 3-D Electromagnetic Wave and all other mysterious Quantum Phenomena. Such structure of a photon or quanta is based on the reality of the nature.

The mass in the nucleus of a spinning photon generates the intrinsic spin angular momentum of continuously changing direction. Wave Particle Duality \& Interference Explained [4] explains few Quantum Phenomena like the Wave Particle Duality, Constructive, Destructive \& Intermediate Interferences and Polarization phenomena.

Similarly, the charge in the nucleus of a spinning photon generates the intrinsic spin 
magnetic dipole moment. The spin angular momentum and the spin magnetic dipole moment bless the spinning \& moving photon with the unique characteristics to display the mysterious behavior.

The electromagnetic force is the second strongest force after the strong force. The electromagnetic fields of the photons/quantum particles interact with the electromagnetic fields of the medium of the conductor, semiconductor or the insulator to display mysterious quantum phenomena. These interactions develop the electromagnetic forces strong enough to change the spin, direction, wavelength \& amplitude etc. of the photons/ quantum particles.

1) Generation of THZ magnetic fields in transparent dielectrics by linearly polarized light

During the experiments in the University of Michigan, W. M. Fischer \& S.C. Rand [5] found a new Quantum Phenomenon "A polarized light of appropriate intensity through a transparent dielectric can generate magnetic effects that are 100 million times stronger". The followings explain this phenomenon in brief as under:

- When a linearly polarized photon of high intensity passes through a transparent high dielectric material, the electric insulating properties of the material prevent the photon from generating the electric field. However, the photon generates the magnetic field depending on the nature of the dielectric.

- The electric field being close to zero, both the amplitude and the wavelength of the EM wave formed by the photon in high dielectric material are also close to zero. However, the frequency of the photon remains the same.

- Due to the negligibly small wavelength and amplitude, the photon generates concentrated magnetic field of high intensity, as the magnetic field cannot spread in $\mathrm{X}$ \& $\mathrm{Z}$ directions due to the negligibly small wavelength \& amplitude. The magnetic field extends in the Y-direction with high intensity as shown in Figure 5(b).

- Due to the electromagnetic field of the high dielectric medium, the photon keeps on spinning with negligibly small wavelength and moves forward at very slow velocity in comparison to its original velocity in the vacuum.

- Every new spin of the photon generates a new magnetic field of very high intensity in the Y-directions. The new high intensity magnetic field is close to the previous magnetic field due to very small wavelength of the polarized photon. Therefore, there are multiple magnetic fields in very close proximity as shown in Figure 5(b).

- All these very high intensity magnetic fields, being close by close, merge together to generate peaks of the magnetic field of several million times strengths of the original strength as shown in Figure 5(c).

- Based on the natures of the polarized photons \& the dielectric material, the magnetic fields of Terahertz can be generated. This quantum phenomenon is shown in Figure 5 pictorially.

\section{2) Quantum tunneling}

In the phenomenon of Quantum tunneling, some of the quantum particles can tunnel through an insulator or a barrier, which otherwise these quantum particles cannot 


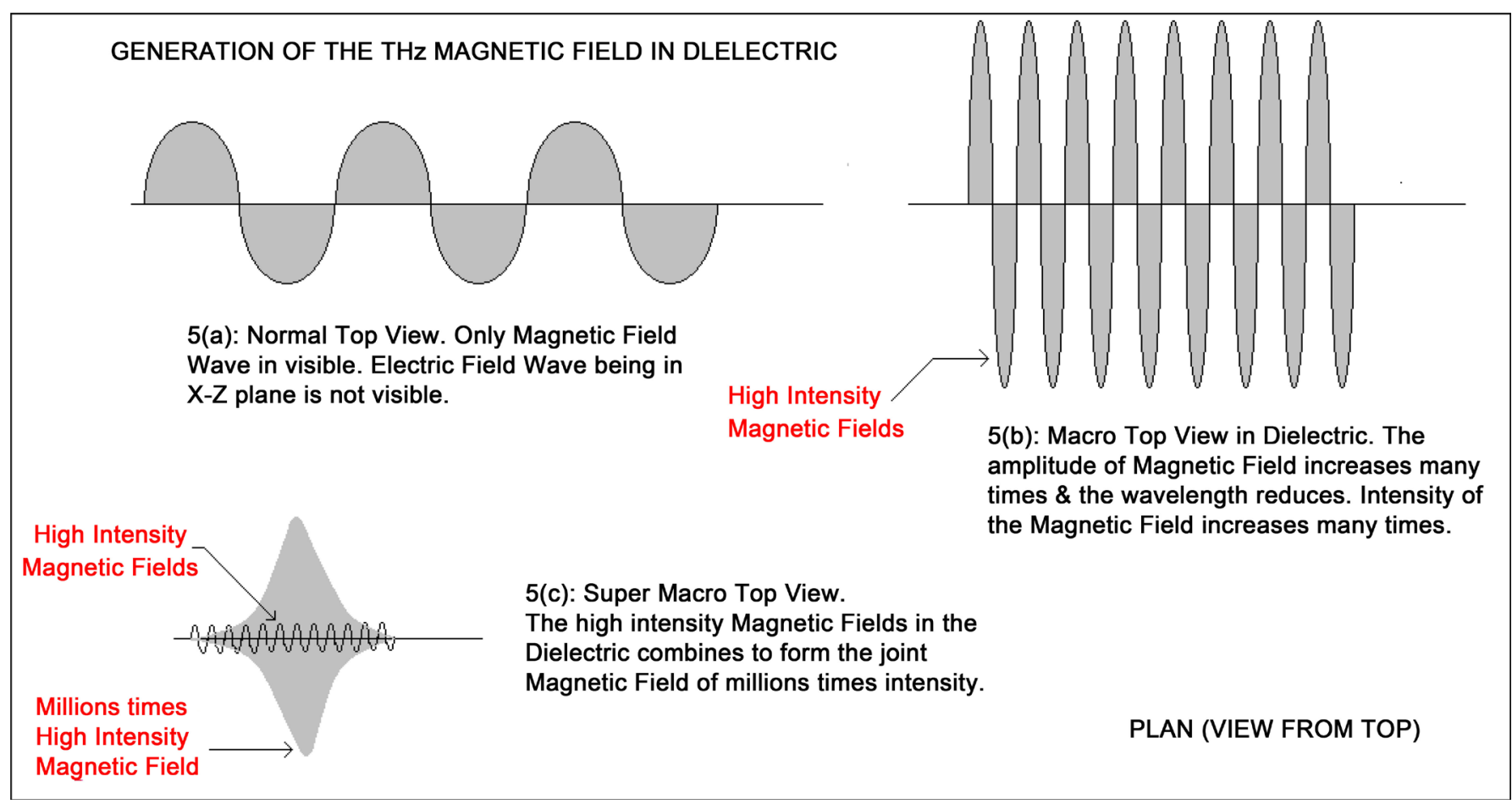

Figure 5. (a) Normal view of polarized light in vacuum. Only the magnetic field is visible, the electric field being only in the vertical $\mathrm{X}-\mathrm{Z}$ plane is not visible. (b) Macro view in dielectric medium. Negligibly small electric field generates in the vertical X-Z plane. High intensity magnetic fields generate with each spin of the photon. (c) Super Macro view in dielectric medium. The High intensity magnetic fields combine to form the peak of the several millions times intensity of the joint magnetic field. All the figures are "Plan View" from the top of Electromagnetic Wave formed by the photon.

surmount. The followings explain this phenomenon in brief as under:

- The quantum particles like electrons/photons move in the path of waves and generate their own electric \& the magnetic fields.

- When unpolarized quantum particles, with the polarization angles distributed in the whole range of $360^{\circ}$ angle, encounter a thin barrier or insulator etc. The electric \& the magnetic fields of both the quantum particles as well as of the barrier interact.

- The electromagnetic field of the barrier reflects back most of the quantum particles as their electromagnetic fields do not match with the electromagnetic field of the barrier. The strong enough electromagnetic field of the barrier changes the spin/direction of the quantum particles to deviate \& ultimately reflect by the barrier.

- However, few quantum particles polarized in specific angles have their electromagnetic fields matching with the electromagnetic field of the barrier.

- The barrier permits only such quantum particles with the matching electromagnetic fields to enter the barrier and pass through the barrier.

- The electromagnetic fields of the quantum particles interact with the electromagnetic field of the barrier. This results in the change in the electric and magnetic fields of the quantum particles during their travel through the barrier.

- There is change in the wavelength, amplitude, direction \& spin etc. of the quantum particles while passing through the barrier. 
- Therefore, only few quantum particles, with matching electromagnetic field, can only pass through the barrier with modified amplitude, wavelength, electric \& magnetic fields. These quantum particles exit the barrier with changed characteristics depending on the nature of the barrier/insulator $\&$ the quantum particles.

\section{3) Hall effect}

Edwin Herbert Hall detected the drift of the electrons in a strip conductor by an external magnetic field in the year 1869. The Hall Effect is the production of a voltage difference transverse to the electric current in an electrical conductor when an external magnetic field perpendicular to the current is applied. This effect is also the result of interactions amongst the electromagnetic fields of the electrons and the external magnetic field. The followings explain this phenomenon in brief as under:

- On applying a voltage to a strip conductor, the electrons start moving in the opposite direction. In a good conductor, the free electrons have easy passage.

- The electric fields of the moving free electrons are in the plane of the direction of the electrons travel. The magnetic fields of the moving free electrons generate in the direction perpendicular to the direction of travel of the electrons.

- When an external magnetic field is applied perpendicular to the strip conductor, the magnetic field of the external magnet influences the electromagnetic fields of the free electrons.

- The magnetic fields of both the moving free electrons \& the external magnet interact to develop force of attraction on the free electrons towards the north pole of the external magnet.

- Simultaneously, repulsive force develops by the external magnetic field from the south pole side of the external magnet.

- This interaction drifts the free electrons towards one side of the strip conductor.

- The continued drifting of most of the electrons, on one side of the conductor, develops the negative charge on one side and the positive charge on the other side of the strip conductor.

- The excessive electrons on one side of the conductor and the less electrons on the other side, develop the electrostatic force across the width of the conductor. This electrostatic force regulates the drifting of the free electrons.

- Soon the equilibrium achieves by the interactions amongst the electromagnetic fields \& the electrostatic force due to the voltage difference. The drifting of the electrons on one side of the conductor stabilizes maintaining a voltage difference across the width of the conductor.

\section{4) Spin hall effect}

The Spin Hall Effect consists in spin accumulation at the lateral boundaries of a current-carrying conductor, the directions of the spins being opposite at the opposing boundaries. M.I. Dyakonov and V.I. Peril [6] in 1971 predicted the Quantum Phenomenon of Spin Hall Effect. It took more than 30 years to observe this quantum phenomenon in the semiconductors experimentally. The followings explain this phenomenon in brief as under: 
- The semiconductors have uniform lattice and a few free electrons. The atoms are satisfied with the covalent bonds with the neighboring atoms. The doping creates free electrons \& holes.

- There are strong electromagnetic forces amongst the shared electrons and the nuclei of the atoms sharing the electrons to hold the electrons in their covalent bonds.

- A shared electron orbits in the external shells of the atoms sharing the electron. This electron has its own electromagnetic field under the influence of electromagnetic fields of the two sharing nuclei and other close by electrons/the nuclei of the other atoms. All these electromagnetic fields vary continuously.

- Being shared electron of a covalent bond, generally the electron does not break loose. However, the continuously varying effects of the multiple electromagnetic fields affect the electromagnetic field of the electron to deviate from its routine path of a covalent bond.

- This changes the direction of the spin of the shared electron to leave the bond and break free. The exit of an electron from its covalent bond creates a hole in its place.

- The hole is filled either by a free electron or by an electron of a covalent bond to create a new hole.

- The above explains the creations of the holes and the free electrons. The process of the formation of free electrons and the holes continues in the semiconductor.

- On applying a voltage across the semiconductor, the process of the formation of free electrons and the holes intensifies throughout the semiconductor.

- The atoms satisfied with covalent bonds generally surround the free electrons and restrict the flow of free electrons even in the presence of a voltage difference. Therefore, the free electrons have to move in a zigzag manner to find the holes to move forward.

- The free electrons, near the edge/surface of the semiconductor, experience less resistance to move towards the edge/surface. Less the number of atoms towards the edge/surface, less is the resistance to the free electrons towards the edge/surface.

- The electrons do prefer to travel by the least resistance path. Therefore the free electrons move towards the edge/surface creating the holes towards the edge/surface.

- This process creates more holes towards the edge/surface of the semiconductor on applying a voltage difference.

- The various electromagnetic fields continuously interact with the electromagnetic fields of the electrons. The electrons, which can orient their spins \& movements in the direction of the holes (more holes are available towards the edge/surface), jump to the holes creating lateral movement of the electrons from the middle of the semiconductor towards the edge/surface due to the interactions of the electromagnetic fields.

- The spin of an electron (a photon or quanta) is always in the direction of its travel. Therefore the electrons move towards the edge/surface with the spin in the direction towards the edge/surface, in order to move forward towards the edge/surface of the semiconductor.

- The opposite surfaces have opposite spins of the electrons due to the movements in 
the opposite directions.

\section{5) Quantum spin hall effect}

Quantum Spin Hall Effect involves spin-orbit interlocking, formation of conical spectrum, vortex spin texture and transverse spin in evanescent waves etc. This quantum phenomenon along with experiment is described in detail by Konstantin Y. Bliokh, Daria Smirnova \& Franco Nori in Quantum Spin Hall Effect of Light [6]. The followings explain Quantum Spin Hall Effect in brief for a circularly polarized light on semiconductor forming a conical spectrum/evanescent wave:

- A circularly polarized photon generates the electric field between the centreline and the circularly curved wave path of the photon in all the $3 \mathrm{X}, \mathrm{Y} \& \mathrm{Z}$ directions. Simultaneously the magnetic field generates perpendicular to the plane of the electric field in all the $3 \mathrm{X}, \mathrm{Y} \& \mathrm{Z}$ directions.

- In the vacuum, the circularly polarized photon moves freely, in its own 3-D path without any external effect, maintaining the spin, direction, wavelength, amplitude and the intensities of the electric \& the magnetic fields.

- When a circularly polarized photon enters a semiconductor, the electromagnetic field of the semiconductor interacts with the electromagnetic field of the photon.

- Depending on the nature of the semiconductor, the electromagnetic field of the semiconductor restricts to reduce the formation of both the electric \& the magnetic fields of the circularly polarized photon.

- This continuously reduces the spin, direction, wavelength, amplitude and the intensities of the electric \& the magnetic fields of the photon. The velocity of the photon in the semiconductor also reduces continuously.

- Due to the continuous reducing effect on amplitude \& wavelength, the circularly polarized photon moves in the path of a conical spectrum/vortex spin texture. The shape of the conical spectrum/vortex spin texture depends on the nature of the semiconductor and the photon.

- The continuous reduction in the wavelength $\&$ amplitude along with the intensities of the electric \& the magnetic fields and the change in the spin $\&$ the direction of the photon results in ultimately zero wavelength $\&$ amplitude as an evanescent wave.

\section{Conclusions}

The photons \& the quantum particles are no ordinary particles since these can display mysterious Quantum Phenomena. The nature has provided a photon or quanta some special characteristics.

According to the New Quantum Theory, a photon or quanta has a nucleus of the mass \& the charge located off-center. The structure of a photon or quanta with the off-centre mass $\&$ the charge is not hypothetical but is common in the nature all around us, in all the atoms.

The mass located off-centre in a spinning \& moving particle generates intrinsic spin angular momentum of continuously changing direction. Similarly, the charge located off-centre in a spinning \& moving particle generates spin magnetic moment of conti- 
nuously changing magnitude \& direction. The continuously varying spin angular momentum \& the spin magnetic moment bless a photon or quanta with capabilities to display mysterious Quantum Phenomena.

This explains not only the long pending Wave Particle Duality \& the formation of 3-D Electromagnetic Wave but all other Quantum Phenomena.

This works proves that a photon has the mass and the charge. The mass of a photon is directly proportional to its frequency. The mass of a photon of any frequency can be calculated simply by multiplying the frequency of the photon with "a" (Mass-frequency constant) having value $1.474499440284 \times 10^{-50} \mathrm{~kg} \mathrm{sec}$. This work also explains some of the Quantum Phenomena in brief.

The electromagnetic fields/spin magnetic moment of the photons/quantum particles interact with the electromagnetic fields of the mediums of the conductor, semiconductor or the insulator to display mysterious Quantum Phenomena. These interactions develop the strong enough electromagnetic forces which change the direction, wavelength, amplitude and spin etc. of the photons or the quantum particles to display mysterious Quantum Phenomena.

This work provides a new direction to investigate, develop \& modify the various Quantum Phenomena for the improvements/new developments for the use in medical, industrial \& commercial applications for the benefit of all.

\section{References}

[1] Selleri, F. (1990) Quantum Paradoxes and Physical Reality. Kluwer Academic Publishers, The Netherlands. http://dx.doi.org/10.1007/978-94-009-1862-7

[2] Agarwal, N.S. (2012) Indian Journal of Science and Technology, 5, 3612-3617.

[3] Agarwal, N.S. (2015) Journal of Modern Physics, 6, 627-633. http://dx.doi.org/10.4236/jmp.2015.65068

[4] Agarwal, N.S. (2016) Journal of Modern Physics, 7, 267-276. http://dx.doi.org/10.4236/jmp.2016.73026

[5] Fisher, W.M. and Rand, S.C. (2011) Journal of Applied Physics, 109, Article ID: 064903. http://dx.doi.org/10.1063/1.3561505

[6] Dyakonov, M.I. and Perel, V.I. (1971) Physics Letters A, 35, 459. https:/doi.org/10.1016/0375-9601(71)90196-4 
Submit or recommend next manuscript to SCIRP and we will provide best service for you:

Accepting pre-submission inquiries through Email, Facebook, LinkedIn, Twitter, etc. A wide selection of journals (inclusive of 9 subjects, more than 200 journals)

Providing 24-hour high-quality service

User-friendly online submission system

Fair and swift peer-review system

Efficient typesetting and proofreading procedure

Display of the result of downloads and visits, as well as the number of cited articles

Maximum dissemination of your research work

Submit your manuscript at: http://papersubmission.scirp.org/

Or contact jmp@scirp.org 